\title{
Proton field-cycling nuclear magnetic resonance relaxometry in the smectic A mesophase of thermotropic cyanobiphenyls: Effects of sonication
}

\author{
F. Bonetto and E. Anoardo a) \\ Facultad de Matemática, Astronomía y Física, Universidad Nacional de Córdoba, Ciudad Universitaria, \\ X5016LAE Córdoba, Argentina
}

(Received 1 December 2003; accepted 22 March 2004)

\begin{abstract}
Proton field-cycling nuclear magnetic resonance relaxometry is used to study the spin-lattice relaxation dispersion of selected standard smectic A liquid crystals at different temperatures. Relaxation features at both, in the presence and absence of a monochromatic ultrasonic field are considered. We show that the laboratory-frame spin-lattice relaxation time is mainly governed by traslational diffusion. Order director fluctuations (ODF) are less important while rotational difussion seems to be only relevant near the clearing point. Our study suggests that sonication enhances the ODF contribution in the SmA mesophase. Within the framework of the approach we have outlined, different features associated with the ODF mechanism can be investigated. (C) 2004 American Institute of Physics. [DOI: 10.1063/1.1740751]
\end{abstract}

\section{INTRODUCTION}

The Larmor frequency $\nu$ dispersion of proton spin-lattice relaxation time $T_{1}$ in thermotropic liquid crystals may be analyzed through the superposition of different competing relaxation mechanisms. Among the most relevant we may consider collective molecular motions like order director fluctuations (ODF), and individual molecular motions such as translational and rotational self-diffusions. It should be emphasized that the smectic A (SmA) mesophase was scarcely studied with the field-cycling technique. Remarkably, some features related to the $T_{1}$ relaxometry of these systems are still left as an open question. A characteristic of these systems is the strong dispersion of the $T_{1}$ appearing in the low-frequency end of the available Larmor frequency window. Such strong dispersion was usually associated with the smectic ODF mechanism. However, it was recently shown that, at least for cyanobiphenyls, such dispersion is more connected with hardware setting and the presence of local fields. ${ }^{1}$ Since we have lost the ideal scenario of a $T_{1}$ relaxation parameter measurable within the local field region, we decided to investigate again the SmA mesophase with the main spirit of disentangling ODF and diffusion contributions.

Two main theoretical models have been proposed up to now for the SmA mesophase. The former, ${ }^{2}$ predicts a linear behavior of $T_{1}$ with Larmor frequency $\nu\left[T_{1}(\nu)=\alpha \nu\right]$. However, this Larmor frequency dependence was estimated from the undulation layer mechanism in the limit where the correlation length $\xi$ does not depend on the wave vector of the hydrodynamic modes $\vec{q}$. If a more realistic relationship $\xi \propto 1 / q^{2}$ is assumed, a logarithmic dependence for $T_{1}(\nu)$ is obtained. A second relaxation model proposed by Vilfan et al. ${ }^{3}$ foresees a behavior for $T_{1}(\nu)$ ranging approximately

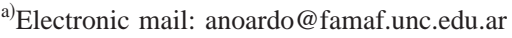

from $\nu^{0}$ at low frequencies (minor than $1 \mathrm{MHz}$ ) up to $\nu^{0.5}$ in the high frequency regime (in logarithmic scale). In a limiting case (when the SmA phase only involves splay deformations and compressions of the layers), the above mentioned logarithmic behavior is reobtained.

The characteristic leveling-off behavior predicted by this last model at low frequencies anticipates the difficulties that may arise in disentangling ODF from diffusion or other noncollective mechanisms. The situation can be partially circumvented by extending the relaxation dispersion study to higher frequencies, outside the technical limits of the field-cycling method (by measuring $T_{1}$ at fixed frequencies using standard pulse sequences). But a final word depends on how strong can be the dominance of individual molecular motions over the collective ones within that extended high frequency range. This last comment warrants a remarkable observation: There is no reason a priori to assume that collective motions are slow, since they may be effective up to frequencies corresponding to the high frequency cutoff (associated with molecular dimensions) within the high $\mathrm{MHz}$ regime. A completely different point is that ODF dominates, for example, the relaxation dispersion of nematogens in the $\mathrm{kHz}$ region.

The typical dispersion of the $T_{1}$ relaxation time experimentally observed in the SmA phase of cyanobyphenyls is characterized by a weak frequency dependence between 1 $\mathrm{MHz}$ and a frequency of about $20 \mathrm{kHz}$. At this frequency a noticeable steep dispersion appears until about $800 \mathrm{~Hz}$, where a low frequency plateau manifests. The strong dispersion was usually explained through order director fluctuations, while the low frequency plateau was interpreted in terms of the low-frequency ODF cut-off mechanism. ${ }^{4-10}$ In most of these works, the simplified model that predicts a linear ODF dispersion was invoked. However, recent studies suggest that the observed steep dispersions are a conse- 
quence of improper hardware setting and data handling, in combination with the higher nonaveraged local fields owing to the smectic state. 1,11

Nematic (N) ODF may be enhanced with the application of an ultrasonic field. ${ }^{12,13}$ This behavior responds to the fact that the ultrasound couples with the director field, leading to changes in the relaxation dispersion. Such changes may be characterized by ${ }^{14}$

a variation on the dispersion exponent: A behavior that ranges from $\nu^{\alpha}$ (for low frequencies) to $\nu^{1 / 2}$ is obtained for $T_{1}(\nu)$, being $\alpha$ bigger or smaller than 0.5 depending on the direction of the acoustic propagation;

(ii) a uniform relaxation enhancement within the observed Larmor frequency window.

The coupling of ultrasound with noncollective motions was found to be negligible within experimental errors (at the working frequency employed in these experiments).

In this work we study the dependence of the $T_{1}$ relaxation dispersion in two different SmA cyanobiphenyls on the absence and presence of ultrasonic waves. Experiments were performed at different temperatures and restricted to those frequencies where the FC technique does not present limitations related to the presence of local fields. Our main goal at the outset is to investigate the observed changes in the dispersions in terms of the ultrasound-ODF coupling. To our knowledge, the present work constitutes the only case where information about smectic ODF mechanism is extracted from the proton field-cycling NMR $T_{1}$ relaxation dispersion in the SmA phase of cyanobiphenyls.

\section{EXPERIMENT}

Commercial samples of 4-octyl-4' -cyanobiphenyl (8CB) and 4-undecyl-4' -cyanobiphenyl (11CB) from Synthon and Merck Chemicals, respectively, were employed. 8CB presents three phase transition: Solid (K)-SmA at $294.5 \mathrm{~K}$, SmA-N at $306.5 \mathrm{~K}$ and $\mathrm{N}$-isotropic (I) at $313.5 \mathrm{~K}$, while $11 \mathrm{CB}$ only displays two phase transitions: $\mathrm{K}-\mathrm{SmA}$ and SmA-I at 326 and $330.5 \mathrm{~K}$, respectively.

A Stelar FC2000 fast field cycling nuclear magnetic resonance (NMR) relaxometer was used to acquire all the proton relaxation dispersion curves. Standard pre-polarized (PP) and non-polarized (NP) sequences $(0.25$ and $0.23 \mathrm{~T}$ as polarization and detection fields, respectively) were used to obtain $T_{1}$ values. ${ }^{15}$ In all cases, sixteen points (four scan each) were used to determine $T_{1}$. Relative errors were found to range between $2 \%$ and $8 \%$.

The ultrasound was transmitted to the sample through a $3 \mathrm{~mm}$ diameter glass sonotrode coupled to a Hielsher UIP50 generator working at $(29.8 \pm 0.5) \mathrm{kHz}$. An efficient power transmission was obtained by directly placing the glass sonotrode into the sample volume. The temperature control was carried out by a Stelar VTC unit connected to a thermocouple located at about $30 \mathrm{~mm}$ from the bottom of the sample. The control unit was previously calibrated in order to display the real sample temperature. In order to ensure the temperature constancy during the whole experiment, the sample temperature was measured before and after each $T_{1}$ dispersion acquisition with a thermocouple directly immersed into the sample. Direct temperature measurement during NMR acquisition might be grossly unfavorable from the standpoint of sensitivity. At our experimental conditions was not feasible due to the high noise introduced by the thermocouple. Absolute errors in temperature measurements were $\pm 0.2 \mathrm{~K}$ for $8 \mathrm{CB}$ and $\pm 0.5 \mathrm{~K}$ for $11 \mathrm{CB}$ (higher temperature gradients according to a major difference between sample and environment temperatures).

Two different ultrasound power levels were used in the experiments: 13.5 and $22.5 \mathrm{~W} / \mathrm{cm}^{2}$. Higher powers were not used to avoid cavitation and significant temperature gradients across the sample.

\section{RELAXATION MODELS}

As it was previously stated, three different relaxation mechanisms were used to explain the $T_{1}(\nu)$ behavior: One of collective nature (ODF) and two of individual nature (translational diffusion and molecular reorientations). By translational diffusion mechanism, or self diffusion, we mean the modulation of the dipole-dipole interaction between protons located on different molecules, as a consequence of the particle motion, usually thermally activated, from one site to another. Reorientations are intended to be the contributions to the spectral densities at the Larmor frequency caused by rotations around the long and short molecular axis, and rotations of the chain groups. Self diffusion was found to be the more important mechanism driving the $T_{1}$ relaxation in the SmA phase. ${ }^{4}$ This assertion was generally checked to be consistent with our results. Therefore, in the following we will refer to self diffusion as the only important individual relaxation mechanism.

Assuming different time scales for the different mechanisms and small cross terms between them at the correlation function level, ${ }^{13}$

$$
\frac{1}{T_{1}}=\frac{a_{1}}{T_{1 O F}}+\frac{a_{2}}{T_{1 T r}},
$$

where $T_{1}^{-1}$ is the total proton spin relaxation rate; $a_{1}$ and $a_{2}$ are constants; and $T_{1 O F}^{-1}, T_{1 T r}^{-1}$, are the relaxation rates produced by ODF and translational diffusion mechanisms, respectively, in the absence of the other mutually relaxation mechanisms.

At this point, it is important to remark that due to the low spectroscopic resolution of the field-cycling experiments, separate contributions from phenyl and chain protons, at least for these compounds, are not distinguishable. Rapid spin diffusion ensures a single average $T_{1}$, as can be concluded from the mono-exponential evolution of the magnetization decay.

As described in the preceding section, there are essentially two proposed models that may be considered to explain the Larmor frequency dispersion due to the ODF relaxation mechanism in the SmA phase:

- The "nematic" approach.

Consider the nematic free energy: 


$$
f_{n}=\frac{1}{2}\left\{K_{11}(\nabla \cdot \vec{n})^{2}+K_{22}(\vec{n} \cdot \nabla \times \vec{n})^{2}+K_{33}[(\vec{n} \cdot \nabla) \vec{n}]^{2}\right\},
$$

where $K_{11}, K_{22}$, and $K_{33}$ are splay, twist and bend elastic constants, respectively, and $\vec{n}$ is the local director.

If the contribution of smectic undulation waves is taken into account while assuming that the correlation length in the direction perpendicular to the smectic layers does not rely on the mode's wave vector, we have ${ }^{2}$

$$
T_{1 O F}^{n e m}(\nu)=C \nu,
$$

where constant $C$ depends on the Boltzmann and Planck constants, the gyromagnetic ratio, temperature $T$, splay elastic constant $K_{11}$, the interproton distance, order parameter $S$, and the coherence length in the direction perpendicular to the smectic layers. The previous supposition is mathematically (but not physically) equivalent to assume that the relation $K_{33} \ll K_{11}, K_{22}$ is satisfied in Eq. (2). ${ }^{16}$

\section{- The "smectic" approach.}

In this model the coupling of smectic order with director fluctuations is taken into account. ${ }^{3}$ Within this picture, smectic $f_{s}$ and the nematic-smectic interaction $f_{I}$ free energy terms are added to nematic-elastic free energy $f_{n}$ to obtain total free energy $f$,

$$
f=f_{n}+f_{s}+f_{I},
$$

with,

$$
\begin{aligned}
& f_{s}=\varepsilon(T)|\psi|^{2}+\lambda(T)|\psi|^{4}+\ldots \\
& f_{I}=\left(\nabla+i q_{s} \overrightarrow{\delta n}\right) \psi^{*} \frac{1}{2 M}\left(\nabla-i q_{s} \overrightarrow{\delta n}\right) \psi .
\end{aligned}
$$

In the last expressions, $\varepsilon$ and $\lambda$ are coefficients in the expansion of $f_{s}$ in powers of $\psi, M$ is a mass tensor with components $M_{\|}$and $M_{\perp}$, along the normal to the layers and perpendicular to them, respectively and $q_{s}=2 \pi / d, d$ the distance between smectic layers and $\psi$, the smectic order parameter $[\psi=|\psi| \exp (i \phi)]$.

Using the expression obtained in Refs. 17 and 18 for the amplitudes of the mean square fluctuations of the director for the $q$ th mode $\left(\left\langle\left|n_{\alpha}(\vec{q})\right|^{2}\right\rangle\right.$; with $\left.\alpha=1,2\right)$ and their corresponding decay times $\left[\tau_{\alpha}(\vec{q})\right]$, it is possible to arrive at

$$
\begin{aligned}
T_{1 O F}^{s m}(\nu)=\beta\left[\frac{1}{\sqrt{\delta}} \eta_{1} K_{11}^{-3 / 2} Y\right. \\
\left.+\frac{1}{\sqrt{K_{33} \delta}} \eta_{2} K_{22}^{-1} \frac{1}{\sqrt{1+\sqrt{1+\left(\frac{2 \pi \nu}{\omega_{s 2}}\right)^{2}}}}\right]^{-1},
\end{aligned}
$$

$$
Y=\frac{B}{\delta} \int_{0}^{1} \frac{d x}{x\left(\frac{B}{\delta}\left(1-x^{2}\right)+\frac{K_{33}}{K_{11}} x^{2}\right)^{3 / 2} \sqrt{1+\sqrt{1+\left(\frac{2 \pi \nu}{\omega_{s 1}}\right)^{2}}}},
$$

where $B=\psi_{0}^{2} q_{s}^{2} / M_{\|}$and $\delta=\psi_{0}^{2} q_{s}^{2} / M_{\perp}\left(\psi_{0}\right.$ is the equilibrium value of the smectic order), $\eta_{\alpha}$ are viscosities, $\omega_{s \alpha}$ $=\delta / \eta_{\alpha}$ with $\alpha=1,2$, and $\beta$ depends on the Planck constant, the gyromagnetic ratio, the interproton distance and molecular order parameter $S$.

Concerning $T_{1 T r}^{-1}$ relaxation rate, Vilfan et al. ${ }^{19}$ extended to $\mathrm{SmA}$ phases the model previously proposed by Torrey ${ }^{20}$ for the I phase. Although Vilfan et al. ${ }^{19}$ showed that for low frequencies the anisotropy of the molecules influences the relaxation rate, differences with Torrey's model in the dispersion behavior become important only when the Larmor frequency $\nu$, varies form $0<2 \pi \nu \tau_{D}<0.3$, where $\tau_{D}$ is the diffusional correlation time. As in our experiments the factor $2 \pi \nu \tau_{D}$ ranges from about $6 \times 10^{-5}$ to $2 \times 10^{-2}$ (only $7 \%$ of the range where both models differ significantly), in this frequency window we can approximate

$$
T_{1 i}(\nu)=\chi T_{1 S m}(\nu),
$$

with an appropriate factor $\chi$. In the last equation, $T_{1 i}(\nu)$ is the expression obtained by Torrey for the I phase and $T_{1 S m}(\nu)$ is the expression obtained by Vilfan and Zumer for SmA phases.

Then, due to the exposed above and for the sake of simplicity, the following equation was used for $T_{1 T r}^{-1}:{ }^{20}$

$$
T_{1 T r}^{-1}=\frac{A_{D}}{\nu^{2}}[j(\alpha, x)+j(\alpha, \sqrt{2} x)],
$$

where,

$$
\begin{aligned}
& j(\alpha, x)=v\left(1-\frac{1}{u^{2}+v^{2}}\right)+\left(v\left(1+\frac{1}{u^{2}+v^{2}}\right)+2\right) e^{-2 v} \\
& \times \cos (2 u)+u\left(1-\frac{1}{u^{2}+v^{2}}\right) e^{-2 v} \sin (2 u), \\
& \left(\begin{array}{l}
u \\
v
\end{array}\right)=\frac{1}{2}\left(\frac{q(1 \mp q)}{\alpha}\right)^{1 / 2}, \\
& q={\frac{\alpha x^{2}}{\left(1+\alpha^{2} x^{4}\right)}}^{1 / 2} .
\end{aligned}
$$

Here, $\alpha=\left\langle r^{2}\right\rangle / 12 b^{2}, x=\sqrt{2 \pi \nu b^{2} / D}$, and $\left\langle r^{2}\right\rangle$ is the mean square jump distance, $b$ is the distance of closest approach, $D=\left\langle r^{2}\right\rangle / 6 \tau_{D}$ is the isotropic self diffusion constant and $A_{D}$ is a constant that depends on spin density $n, D, \alpha$, and $b$.

As it was previously stated by Torrey, in the limit of small jump width $\left(\left\langle r^{2}\right\rangle \ll b^{2}\right)$ and low correlation times $\left(\omega \tau_{D} \ll 1\right)$, a simplified version of the Torrey's model is obtained. In this limit, $u=v=x / 2$, and

with 


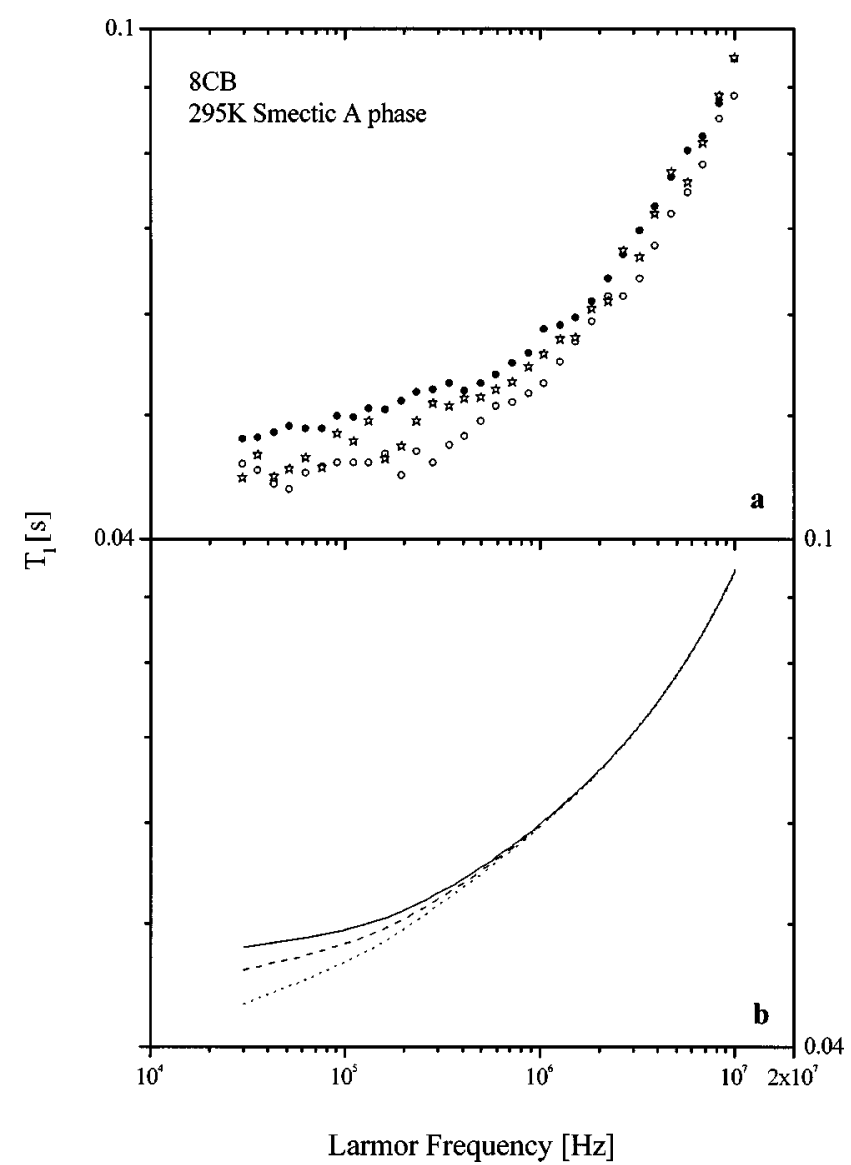

FIG. 1. Relaxation dispersions (a) and simulated curves (b) for 8CB liquid crystal at the SmA phase temperature of $295 \mathrm{~K}$. The three data dispersions and their correlative simulated curves correspond to the three cases: Absence of sound (filled circles and solid line) and presence of it at two different powers $\left(P^{\prime}\right.$ : Hollow stars and dashed line, and $P^{\prime \prime}=22.5 \mathrm{~W} / \mathrm{cm}^{2}$ : Hollow circles and dotted line).

$$
\begin{aligned}
j(\alpha, x)= & \frac{x}{2}-\frac{1}{x}+\frac{x}{2} e^{-x}\left(\left(1-\frac{2}{x^{2}}\right) \sin (x)\right. \\
& \left.+\left(1+\frac{4}{x}+\frac{2}{x^{2}}\right) \cos (x)\right)
\end{aligned}
$$

As it can be observed, this result no longer depends on $\alpha$.

Although hardly justified, this approximated model was broadly used in the analysis of field cycling dispersion curves in liquid crystals in the past. ${ }^{4-10}$ In the present work, the above simplified model was checked to be inappropriate because of the $\tau_{D}$ 's obtained by the corresponding fittings do not satisfy the hypothesis of low correlation times $\left(\omega \tau_{D}\right.$ $\ll 1$ ) assumed by the model in the whole $\omega$ spectrum.

\section{A. Relaxation in the presence of ultrasound}

Ultrasound couples with ODF causing changes in the whole spectrum of the relaxation dispersion curve. ${ }^{12,13}$ In this case, a new orienting term is added to the nematic free energy expression [Eq. (2)]. This term is given by ${ }^{12,21}$

$$
f_{a}=\frac{1}{2} a^{2}(\hat{s} \cdot \hat{n}),
$$

where $a^{2}$ depends on the acoustic intensity, the ultrasound velocity, the average of the sample density, the magnitude of

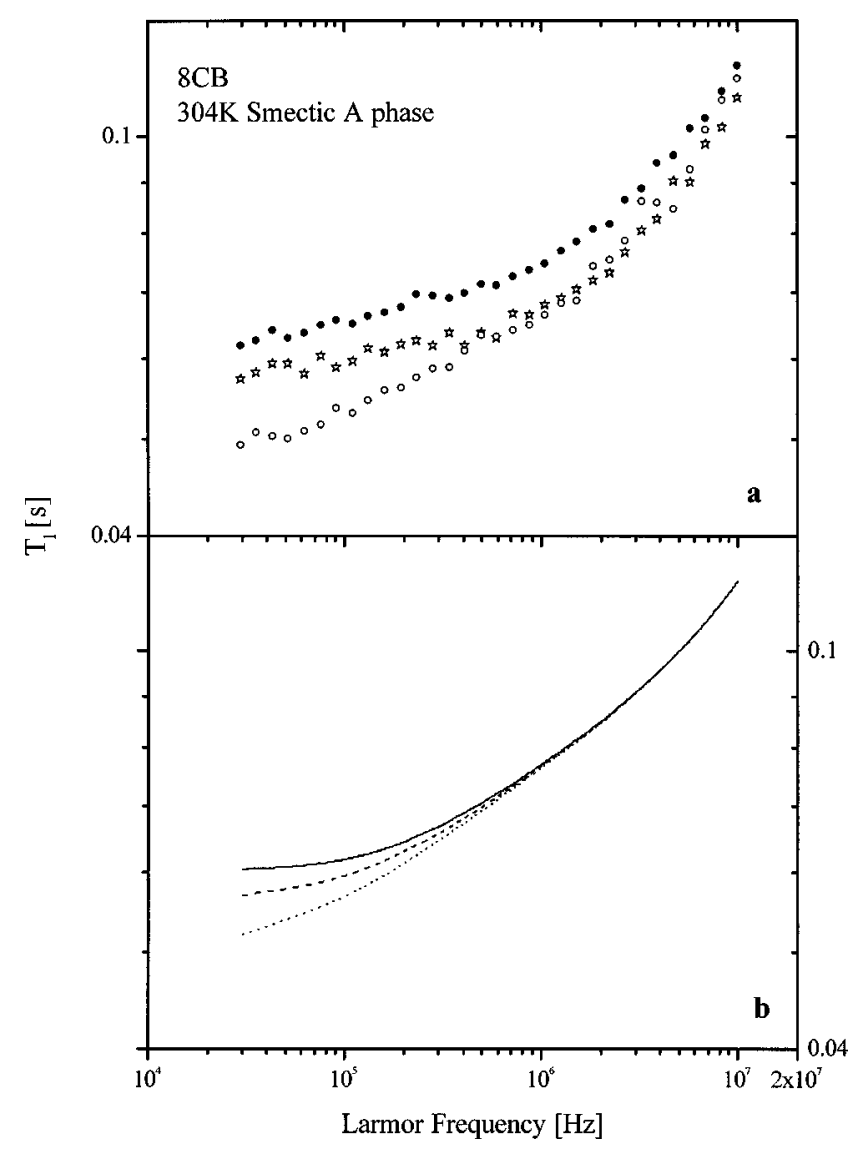

FIG. 2. Relaxation dispersions (a) and simulated curves (b) for 8CB liquid crystal at the SmA temperature of $304 \mathrm{~K}$. The three data dispersions and their correlative simulated curves correspond to the absence of sound (filled circles and solid line) and presence of ultrasonic waves at two different powers: $P^{\prime}=13.5 \mathrm{~W} / \mathrm{cm}^{2}$ (hollow stars and dashed line), and $P^{\prime \prime}$ $=22.5 \mathrm{~W} / \mathrm{cm}^{2}$ (hollow circles and dotted line).

the ultrasound wave vector and the director-density coupling. In the last equation, $\hat{s}$ is an unitary vector pointing along the ultrasonic wave vector, and $\hat{n}$ is the director.

Starting from this new free energy expression, the following is obtained for both, nematic and smectic approaches, in the presence of ultrasonic waves parallel to the director [the detailed calculation is offered in Ref. 14]:

$\begin{aligned} T_{1 O F+S}^{n e m}(\nu)= & \left\{\frac{3 K_{B} T}{4 \pi^{2} \omega} \frac{q_{z c}}{K_{11}} \frac{1}{\sqrt{1+\frac{1}{\left(\omega \tau_{0}\right)^{2}}}}\right. \\ & \left.\left.\times 2 \arctan \left(\frac{\frac{1}{\omega \tau_{0}}}{\sqrt{1+\frac{1}{\left(\omega \tau_{0}\right)^{2}}}}\right)+\pi\right]\right\}^{-1},\end{aligned}$

$$
T_{1 O F+S}^{s m}(\nu)=\beta\left[\frac{1}{\sqrt{D}} \eta_{1} K_{11}^{-3 / 2} Y+j_{1,2}^{O F+S}(\omega)\right]^{-1},
$$

with 


$$
\begin{aligned}
& Y=\frac{B}{D} \int_{0}^{1} \frac{d x}{\left(\frac{B}{D}\left(1-x^{2}\right)+\frac{K_{33}}{K_{11}} x^{2}\right)^{3 / 2} \sqrt{x^{2}-\frac{a^{2}}{D}+\sqrt{\left(x^{2}-\frac{a^{2}}{D}\right)^{2}+\left(\frac{\omega}{\omega_{s 1}}\right)^{2}}}}, \\
& j_{1,2}^{O F+S}(\omega)=\left\{\begin{array}{ll}
\frac{\eta_{2}}{K_{22} K_{33}^{1 / 2} \sqrt{\left|D-a^{2}\right|}} \frac{1}{\sqrt{\sqrt{1+\left(\frac{\omega}{\omega_{s 2}}\right)^{2}}-1}} & \text { if } D \leqslant a^{2} \\
\frac{\eta_{2}}{K_{22} K_{33}^{1 / 2} \sqrt{\left|D-a^{2}\right|}} \frac{1}{\sqrt{\sqrt{1+\left(\frac{\omega}{\omega_{s 2}}\right)^{2}+1}}} & \text { if } D \geqslant a^{2}
\end{array}\right. \text {. }
\end{aligned}
$$

In Eqs. (13)-(16), $\tau_{0}=\eta / a^{2}, \omega_{s 2}=\left|D-a^{2}\right| / \eta_{2}$ and $\omega_{s 1}$ $=D / \eta_{1}$.

Based on experimental facts, ${ }^{12,13}$ it will be assumed that translational self-diffusion relaxation mechanism is not affected by the presence of ultrasonic waves.

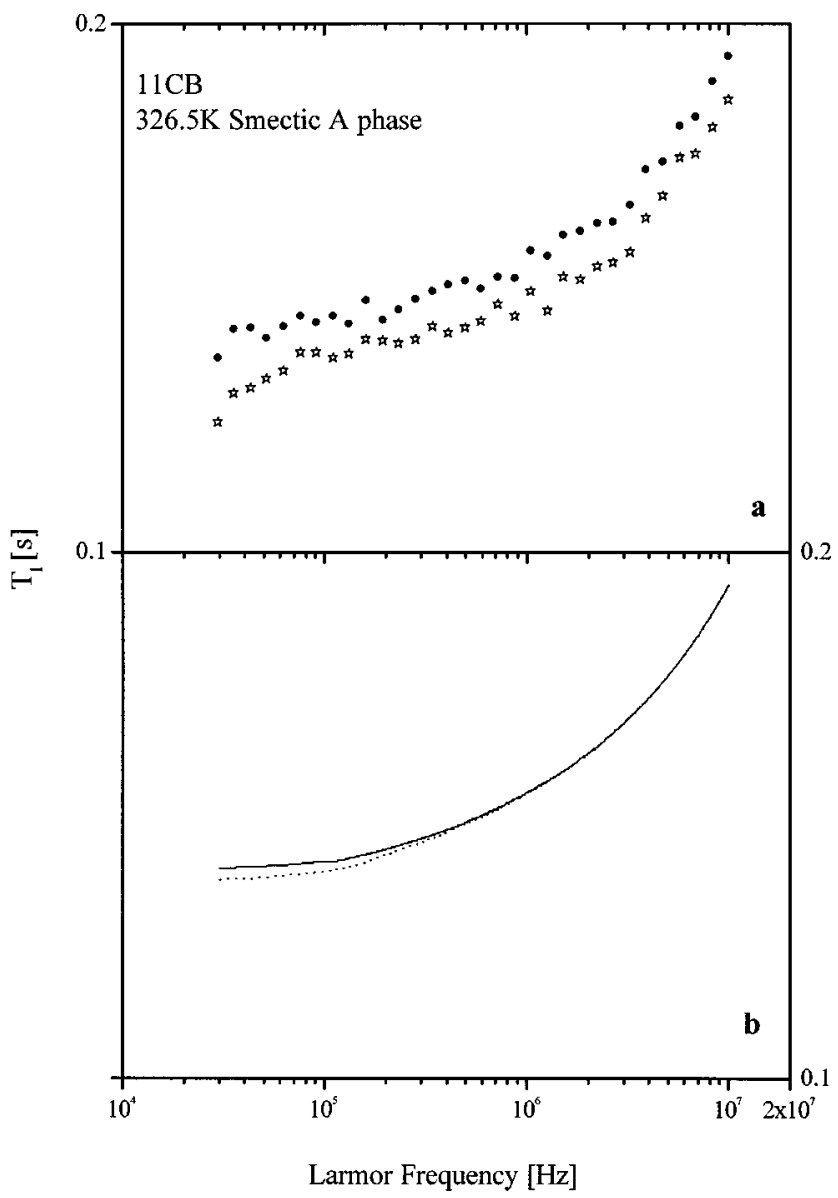

FIG. 3. Relaxation dispersions (a) and simulated curves (b) for 11CB liquid crystal at the SmA temperature of $326.5 \mathrm{~K}$. A clear shift between both measured relaxation profiles (sonicated and nonsonicated) is observed. The two dispersion data and their simulated curves correspond to the absence of sound case (filled circles and solid line) and to the presence of it (at a power $P^{\prime}=13.5 \mathrm{~W} / \mathrm{cm}^{2}$ : Hollow stars and dashed line).

\section{RESULTS}

Figures 1 and 2 show the $T_{1}$ dispersion plots corresponding to a sample of $8 \mathrm{CB}$ at temperatures of 295 and $304 \mathrm{~K}$ within the SmA phase, respectively. Figures 1(a) and 2(a)

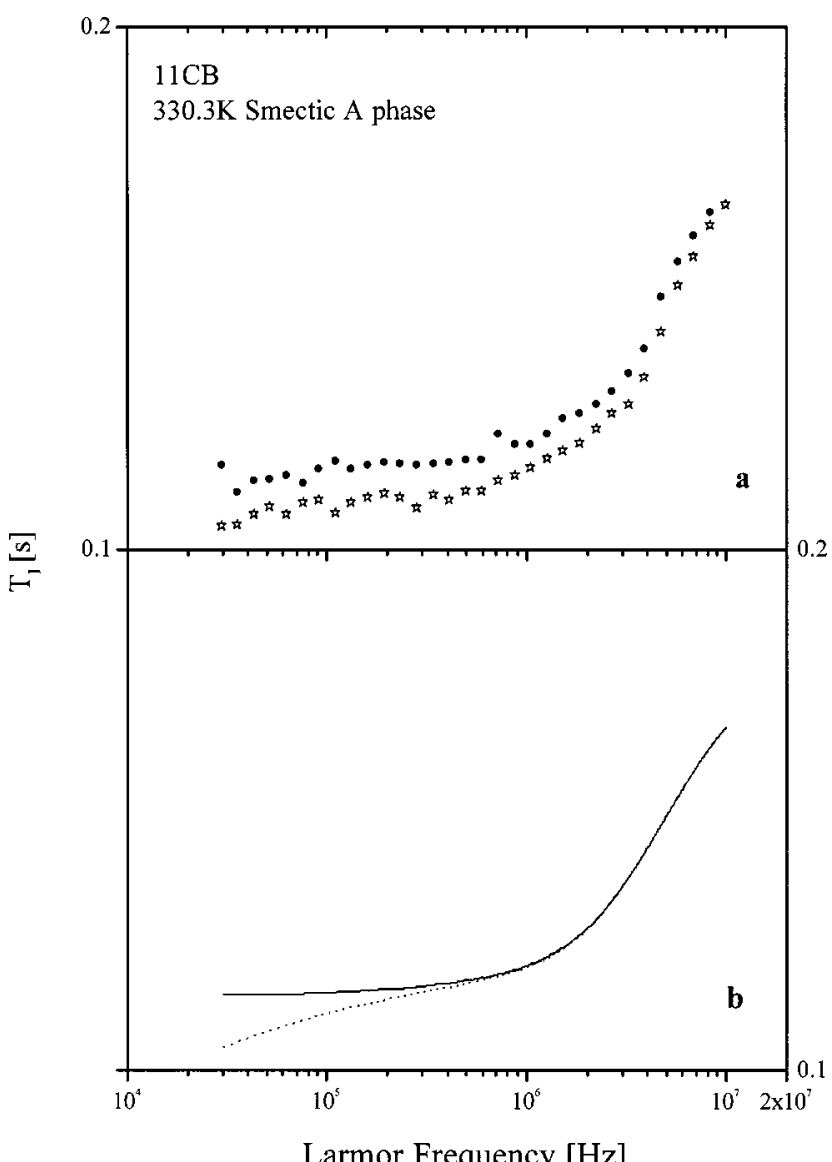

FIG. 4. Relaxation dispersions (a) and simulated curves (b) for 11CB liquid crystal at the $\mathrm{SmA}$ temperature of $330.3 \mathrm{~K}$. As the temperature is near the SmA-I phase transition, rotational relaxation mechanism becomes important. The two data dispersions and their correlative simulated curves correspond to the two cases: Absence of sound (filled circles and solid line) and presence of it at a power of $P^{\prime}=13.5 \mathrm{~W} / \mathrm{cm}^{2}$ (hollow stars and dashed line). 
TABLE I. Parameters used in the simulated curves. $a^{\prime}$ and $a^{\prime \prime}$ stand for the parameter $a$ with ultrasonic powers $P^{\prime}=13.5 \mathrm{~W} \mathrm{~cm}^{-2}$ and $P^{\prime \prime}=22.5 \mathrm{~W} \mathrm{~cm}^{-2}$, respectively. For $11 \mathrm{CB}$ only one ultrasonic power $\left(P^{\prime \prime}\right)$ was employed.

\begin{tabular}{cccccccc}
\hline \hline$B\left[\mathrm{Nm}^{-2}\right]$ & $D\left[\mathrm{Nm}^{-2}\right]$ & $K_{11}[\mathrm{~N}]$ & $K_{22}[\mathrm{~N}]$ & $K_{33}[\mathrm{~N}]$ & $\eta\left[\mathrm{Nsm}^{-2}\right]$ & $a^{\prime 2}\left[\mathrm{Nm}^{-2}\right]$ & $a^{\prime \prime 2}\left[\mathrm{Nm}^{-2}\right]$ \\
\hline $10^{6}$ & $10^{5}$ & $10^{-11}$ & $0.7 \times 10^{-11}$ & $10^{-12}$ & 0.1 & $2.5 \times 10^{4}$ & $5 \times 10^{4}$ \\
\hline \hline
\end{tabular}

show the data obtained in the absence of ultrasonic waves (filled circles) and in the presence of them: At a power of $13.5 \mathrm{~W} / \mathrm{cm}^{2}$ (hollow stars) and at a power of $22.5 \mathrm{~W} / \mathrm{cm}^{2}$ (hollow circles). Figures 1(b) and 2(b) show the corresponding simulated curves for the nonsonicated case (solid line) and in the presence of ultrasonic waves (dashed and dotted lines). Figures 3 and 4 show the corresponding data and simulated curves for $11 \mathrm{CB}$ at smectic temperatures of 326.3 and $330.3 \mathrm{~K}$, respectively.

In the absence of sound, if the linear dispersion model ( $\mathrm{N}$ approach) is assumed to be correct, fittings using Eqs. (1) and (3) indicates that the self-diffusion mechanism completely determines the $T_{1}(\nu)$ behavior in all curves, i.e., the presence of the ODF relaxation mechanism cannot be detected from the measured dispersion curves. This may be the case if the mechanism is masked by self-diffusion. But if this argument was correct, there would not be any difference between dispersion curves in the presence and absence of ultrasound, unless the ultrasonic field was able to pull-up the ODF contribution at considerable strength. In addition, the observed behavior in the presence of sonication cannot be explained through fittings using the nematic approach [Eq. (13) for the ODF contribution]. On the contrary, if the smectic character of the mesophase is added, the behavior obtained is qualitatively well described, as can be seen in Figs. $1-4$.

Simulated curves (except for $11 \mathrm{CB}$ at $330.3 \mathrm{~K}$ ) were obtained through Eq. (1), where (14) was used for $T_{1 O F}$. Due to the high quantity of involved parameters and the complexity of the function, typical values of constants $B, D$, $K_{11}, K_{22}, K_{33}, \eta$, and $a^{2}$ were used to generate simulated curves instead of data fitting (see Table I). ${ }^{3}$ Also, $\alpha$ and $\tau_{D}$ values were fixed (curves without sound were fitted without ODF contribution in order to obtain these approximated values). ${ }^{3}$ Other parameters were fitted, and they can be appreciated in Table II.
In the particular case of $11 \mathrm{CB}$ at $330.3 \mathrm{~K}$, it was found that individual motions were governed by rotations. Then, the simulated curves were obtained following the equation:

$$
\frac{1}{T_{1}}=\frac{b_{1}}{T_{1 O F}}+\frac{b_{2}}{T_{1 \text { Rot }}}+C_{D},
$$

where $^{22}$

$$
\frac{1}{T_{1 \text { Rot }}}=A_{R}\left[\frac{\tau_{R}}{1+\left(2 \pi \nu \tau_{R}\right)^{2}}+\frac{4 \tau_{R}}{1+\left(4 \pi \nu \tau_{R}\right)^{2}}\right]^{-1}
$$

being $A_{R}$ a constant, $\tau_{R}$ the correlation time for rotations, and $C_{D}$ a constant that represents the translational diffusion relaxation mechanism. This result is a consequence of the typical $\tau_{D}$ low values near the SmA-I phase transition $\left(\tau_{D}\right.$ $\sim 10^{-11} \mathrm{~s}$, in most compounds). Then, $2 \pi \nu \tau_{D} \ll 1$ in the whole Larmor frequency range of the measurements, giving a constant behavior for $T_{1 T r}^{-1}$.

Figures 5(a) and 5(b) show how this relaxation mechanism becomes important in the I phase of $8 \mathrm{CB}$ and $11 \mathrm{CB}$ samples, respectively (it is not possible to explain the relaxation in the I phase by using only self-diffusion). In these cases, a non-dispersive contribution for translational selfdiffusion mechanism was found $\left(T_{1 T r}^{-1}=C_{D}\right)$.

\section{DISCUSSION}

Considering we are comparing different compounds, it is important to note that $\tau_{D}$ and $\tau_{R}$ values obtained in the present work show a reasonable agreement with previous values obtained in the smectic phase of terephtahal-bisp-butylaniline (TBBA) ${ }^{5}$ Another important fact is that the $\alpha$ values obtained in the smectic phase for both compounds exhibit the same order of magnitude as the one obtained by Vilfan et al. in TBBA $\left(\left\langle r^{2}\right\rangle / a^{2}=0.04\right) .{ }^{19}$

$T_{1}$ relaxation dispersion data are consistent with a model where individual motions are the dominant relaxation mechanisms. It was found that ODF relaxation dispersion relative weight for $8 \mathrm{CB}$ ranges from $3 \%$ to $9 \%$ at higher

TABLE II. Parameters obtained for $8 \mathrm{CB}$ and 11CB. Relative errors on parameters $\alpha, \tau_{D}$, and $\tau_{R}$ are estimated

\begin{tabular}{|c|c|c|c|c|c|c|c|}
\hline$T[\mathrm{~K}]$ & $A_{D}\left[10^{15} \mathrm{~s}^{-1}\right]$ & $A_{R}\left[10^{6} \mathrm{~s}^{-1}\right]$ & 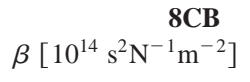 & $C_{D}\left[\mathrm{~s}^{-1}\right]$ & $\alpha\left[10^{-2}\right]$ & $\tau_{D}\left[10^{-10} \mathrm{~s}\right]$ & $\tau_{R}\left[10^{-8} \mathrm{~s}\right]$ \\
\hline 295 & $4.3 \pm 0.9$ & $\cdots$ & $1.4 \pm 0.2$ & $\cdots$ & 1 & 2.4 & $\cdots$ \\
\hline 298 & $5.5 \pm 0.7$ & $\cdots$ & $1.6 \pm 0.8$ & $\cdots$ & 1 & 2.0 & $\cdots$ \\
\hline 301 & $7 \pm 1$ & $\cdots$ & $1.5 \pm 0.2$ & $\cdots$ & 1 & 1.6 & $\cdots$ \\
\hline 304 & $6.5 \pm 1$ & $\cdots$ & $1.2 \pm 0.2$ & $\cdots$ & 1 & 1.6 & $\cdots$ \\
\hline 326 & $\cdots$ & $5.0 \pm 0.3$ & $\cdots$ & $6.2 \pm 0.4$ & $\cdots$ & $\cdots$ & $1.6 \pm 0.2$ \\
\hline \multicolumn{8}{|c|}{$11 \mathrm{CB}$} \\
\hline 326.5 & $6.5 \pm 0.6$ & $\cdots$ & $17 \pm 3$ & $\cdots$ & 2 & 2.5 & $\cdots$ \\
\hline 330.3 & $\cdots$ & $2.2 \pm 0.3$ & $2.0 \pm 0.4$ & $5.8 \pm 0.6$ & $\ldots$ & $\cdots$ & 2.1 \\
\hline 340.0 & $\cdots$ & $2.8 \pm 0.2$ & $\cdots$ & $6.2 \pm 0.4$ & $\cdots$ & $\cdots$ & $0.95 \pm 0.09$ \\
\hline
\end{tabular}
to be of the order of $10 \%$. 


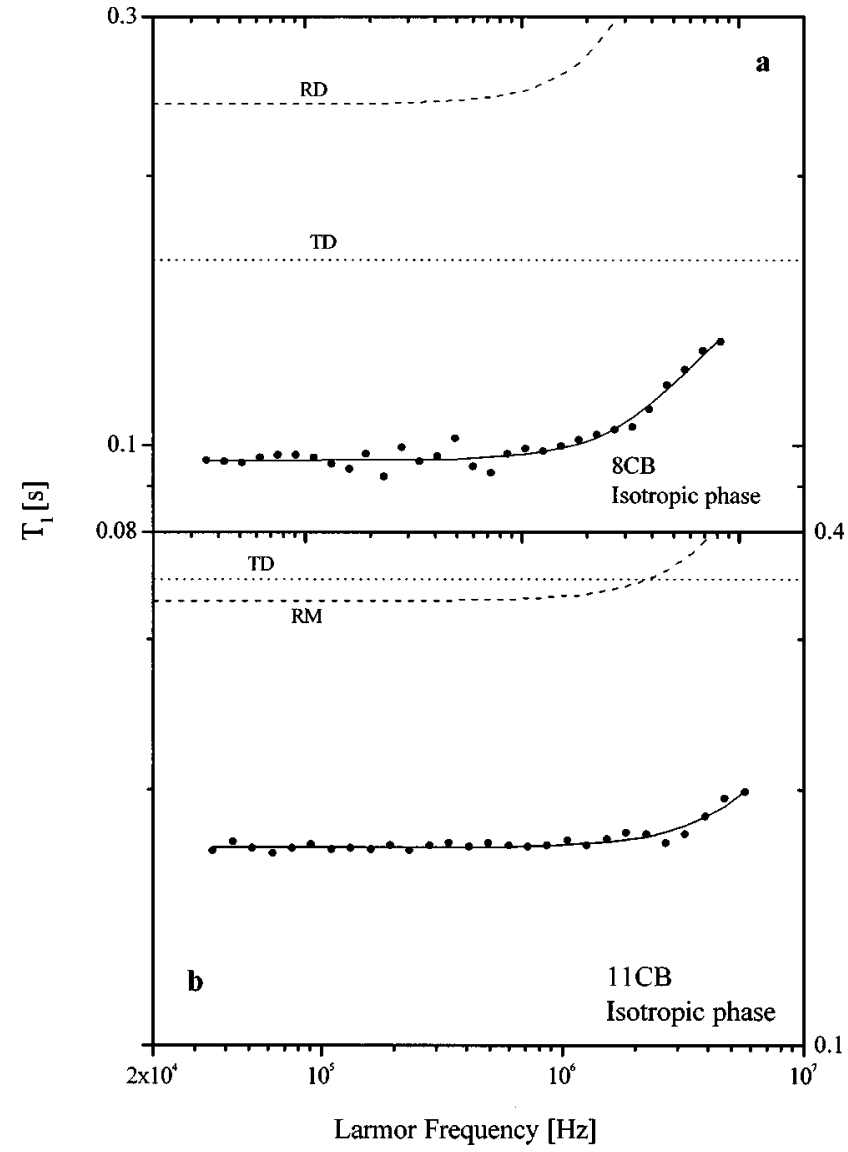

FIG. 5. Relaxation dispersion and fitting curves for 8CB (a) and 11CB (b) in the absence of sound at the I phase temperatures of 326 and $340 \mathrm{~K}$, respectively. It is possible to observe the contribution of rotational diffusion (RD) and translational diffusion (TD) mechanisms in both $T_{1}(\nu)$ dispersions.

frequencies and from $15 \%$ to $25 \%$ at lower frequencies. For $11 \mathrm{CB}$ these values are significantly lower at $326.5 \mathrm{~K}(1,8 \%$ and $8 \%$ at higher and lower frequencies, respectively). This result is consistent with the relative ratio $n_{c} / n_{t}$ (where $n_{c}$ is the number of protons in the core and $n_{t}$ is the number of protons in the tail). ${ }^{13,23}$ It was also found that this relative weight does not present a strong dependence with temperature.

Even though a qualitative agreement is clearly observed between results and the proposed model, there is an effect observable in the experimental data and not explained by the model: The ODF contribution to the relaxation dispersion under sonication is shifted to lower relaxation time values more noticeable in Fig. (3). As it was mentioned above, this effect was already observed in nematics, ${ }^{13}$ and can be attributed to a change in parameter $\beta$ induced by the sound (assumed constant in the model). Physically, this change is related to a possible variation in macroscopic parameters (like viscosity or elastic constants, for example). It is important to mention that this effect was not taken into account in the generation of the simulated curves.

Rotational diffusion only contributes in the SmA phase of $11 \mathrm{CB}$ close to the SmA-I transition. In $8 \mathrm{CB}$ contribution from this mechanism is absent, even at temperatures close to the SmA-N transition. This result can be explained by the presence of pre-transitional disorder caused by the proximity to the SmA-I transition. This transition does not take place in $8 \mathrm{CB}$.

The similitude of the frequency dependence presented by ODF and individual relaxation mechanisms at low frequencies does not allow us to determine the relative weight of each other from the dispersion curves in the absence of ultrasound. The change in the dispersion curve under sonication allow us to identify the presence and the relative weight of ODF in the dispersions.

\section{CONCLUSIONS}

In this work we have shown that the $T_{1}(\nu)$ dispersion in the SmA phase of cyanobiphenyls is consistent with a model where translational diffusion is the dominant mechanism. We also have shown that rotational diffusion starts to be relevant close to the SmA-I phase transition, possibly being a consequence of that.

The measurement of relaxation dispersion with the sample subjected to ultrasonic irradiation allows detecting the presence of the ODF mechanism and quantifying its relative weight. Two previously proposed models were used to explain these data. It was found that a relaxation model including particular features of the smectic phase ${ }^{3}$ can be better used to describe the observed experimental data.

\section{ACKNOWLEDGMENTS}

F.B. acknowledges to CONICET, Fundación Antorchas and Deutsche Akademischer Austauschdienst (DAAD) for financial assistance. E.A. is fellow of CONICET. Financial aid from Secyt-UNC is also acknowledged. The authors would like to express their deep appreciation to Professor Rainer Kimmich (University of Ulm, Germany) for allowing the experimental work at his lab and for permanent support.

${ }^{1}$ E. Anoardo, F. Bonetto, and R. Kimmich, Phys. Rev. E 68, 022701 (2003).

${ }^{2}$ R. Blinc, M. Luzar, M. Vilfan, and M. Burgar, J. Chem. Phys. 63, 3445 (1975).

${ }^{3}$ M. Vilfan, M. Kogoj, and R. Blinc, J. Chem. Phys. 86, 1055 (1987).

${ }^{4}$ T. Mugele, V. Graf, W. Wölfel, and F. Noack, Z. Naturforsch. A 35, 924 (1980).

${ }^{5}$ F. Noack, M. Notter, and W. Weiss, Liq. Cryst. 3, 907 (1988).

${ }^{6}$ D. Pusiol and F. Noack, Liq. Cryst. 5, 377 (1989).

${ }^{7}$ D. Pusiol, R. Humpfer, and F. Noack, Z. Naturforsch. A 47, 1105 (1992).

${ }^{8}$ R. H. Acosta, D. J. Pusiol, Phys. Rev. E 60, 1808 (1999).

${ }^{9}$ R. H. Acosta and D. Pusiol, Phys. Rev. E 63, 011707 (2000).

${ }^{10}$ A. Carvalho, P. J. Sebastião, A. C. Ribeiro, H. T. Nguyen, and M. Vilfan, J. Chem. Phys. 115, 10484 (2001).

${ }^{11}$ E. Anoardo and R. Kimmich, XXXII National Congress on Magnetic Resonance GIDRM, Pavia-Italy (2002).

${ }^{12}$ F. Bonetto, E. Anoardo, and R. Kimmich, Chem. Phys. Lett. 361, 237 (2002).

${ }^{13}$ F. Bonetto, E. Anoardo, and R. Kimmich, J. Chem. Phys. 118, 9037 (2003).

${ }^{14}$ F. Bonetto and E. Anoardo, Phys. Rev. E 68, 021703 (2003).

${ }^{15}$ E. Anoardo, G. Galli, and G. Ferrante, Appl. Magn. Reson. 20, 365 (2001).

${ }^{16}$ R. Y. Dong, Nuclear Magnetic Resonance of Liquid Crystals (Springer, New York, 1997), pp. 146-155. 
${ }^{17}$ F. Brochard, J. Phys. (Paris) 34, 411 (1973).

${ }^{18} \mathrm{R}$. Schaetzing and J. Litster, Advances in Liquid Crystals (Academic, New York, 1979), Vol. 4, p. 147.

${ }^{19}$ M. Vilfan and S. Zumer, Phys. Rev. A 21, 672 (1980).

${ }^{20}$ H. C. Torrey, Phys. Rev. 92, 962 (1953).
${ }^{21}$ J. Selinger, M. Spector, V. Greanya, B. Weslowsky, D. Shenoy, and R. Shashidhar, Phys. Rev. E 66, 051708 (2002).

${ }^{22}$ A. Abragam, Principles of Nuclear Magnetism (Clarendon, Oxford, 1961), pp. 298-300.

${ }^{23}$ K. H. Schweikert and F. Noack, Mol. Cryst. Liq. Cryst. 212, 33 (1992). 